\title{
Tecnología de aplicación terrestre para el control de insectos en el cultivo de soja
}

\author{
Juan J. Olivet ${ }^{1}$, Carlos D. Picos ${ }^{1}$, Juana Villalba ${ }^{2} \&$ Stella Zerbino $^{3}$
}

\begin{abstract}
RESUMEN
Se realizaron dos ensayos para evaluar la eficiencia de deposición y el control de insectos con diferentes métodos de aplicación en el cultivo de soja en Uruguay. En el primer ensayo se estudió el efecto del tamaño de gotas, velocidad de aplicación y horario de aplicación en el control de epinotia (Epinotia aporema W.) El segundo ensayo se dedicó al efecto del tamaño de gotas y la velocidad de aplicación en el control de chinches (Nezara viridula L. y Piezodorus guildinii W.). Se evaluó la distribución de un trazador fluorescente y conteo sobre el follaje. La densidad de impactos fue mayor con gotas finas y medias que con gotas muy gruesas en el haz en ambos estratos, en el envés la densidad de impactos fue similar entre tamaños de gota. En el estrato medio el aumento de la velocidad produjo disminución de la densidad de impactos. En ese estrato, los impactos en el envés fueron muy escasos. El control de epinotia y chinches fue mayor en las parcelas tratadas que en el testigo sin tratar, sin embargo, los diferentes tamaños de gota, velocidad y horario de aplicación tuvieron un control similar. Se destaca la conveniencia de utilización de gotas muy gruesas y medias en el control de insectos en soja por su eficacia y potencial de disminución de la deriva.
\end{abstract}

Palabras-claves: chinches, lagartas, tamaño de gota, velocidad de aplicación

\section{Application of terrestrial technology for control of insects in soybean crop}

\begin{abstract}
Two trials were conducted for efficiency of spray-deposit distribution and for insect control in soybean crop in Uruguay. In the first one the effects of droplet size, volume application rate and application time in the control of bean shoot borer (Epinotia aporema W.) were evaluated. The second trial consisted in evaluating the effects of droplet size and application speed in the control of shield bugs (Nezara viridula L. and Piezodorus guildinii W.). Distribution of a fluorescent dye was evaluated by counting impact on the foliage in the middle and upper canopy. Impact density was higher with fine and medium compared to very coarse droplets on the upper side of the leaves in both trials, but on the inner side, impact density was similar among the three evaluated droplet sizes. Increasing application speed decreased impact density, particularly at the middle height of the foliage. At this height, impact on the underside of leaves was extremely low. Insect control was higher in treated than untreated plots, however, different droplet size, speed and application time resulted in similar control. The results emphasize convenience of very large and medium droplets in the control of insects in soybean due to its efficacy and drift potential reduction.
\end{abstract}

Key words: shield bugs, bud shoot borer, droplet size, application speed 


\section{INTRODUCCIÓN}

En Uruguay, al igual que en otros países de la región el cultivo de soja ha tenido un vertiginoso aumento habiendo alcanzado aproximadamente un millón de hectáreas sembradas en la temporada 2010-2011. Desde al año 2000 se constata un aumento del uso de fitosanitarios de 7 a 20 millones de $\mathrm{kg}$ y las consecuentes preocupaciones por el posible impacto sobre el ambiente y las poblaciones rurales.

La investigación en tecnología de aplicación en el cultivo de soja es amplia, aún así, los resultados suelen ser aparentemente contradictorios. La principal coincidencia entre ellos es que la deposición en la parte media o inferior del follaje resulta bastante reducida en comparación a la deposición en el estrato superior. Antuniassi et al. (2004) evaluando la cobertura de hojas de soja en aplicaciones terrestres determinó que independientemente del volumen aplicado la mayor parte del caldo quedó retenido en el estrato superior el cual llegó a tener hasta diez veces más cobertura que el estrato inferior. Reducciones similares en cuanto a deposición en la parte media e inferior del cultivo fueron obtenidas por diversos autores como Boschini et al. (2008), Hanna et al. (2009), Barbosa et al. (2009), entre otros.

En cuanto al tipo de boquilla y tamaño de gotas, no se registran tantas coincidencias. Para Antuniassi et al. (2004) las boquillas que produjeron gotas finas propiciaron mejor cobertura en soja en los estratos medio y bajo, siendo la menor cobertura obtenida con la boquilla de abanico plano de aire inducido de gotas muy gruesas. Galvez et al. (2005) mediante el uso de tarjetas hidrosensibles en soja observan que la penetración de las boquillas de cono hueco fue mayor que con boquillas de abanico plano simple, doble y de cono lleno. Sin embargo, las diferencias a favor de las de gotas más finas no son tan evidentes cuando la evaluación se realiza mediante técnicas de recuperación. Boschini et al. (2008) mediante la recuperación de un trazador en soja con diferentes boquillas encuentra que la boquilla de cono hueco presentó mayor deposición en el estrato inferior y medio, pero esas diferencias solo fueron claras con tasa de aplicación de 200 y $300 \mathrm{~L} \mathrm{ha}^{-1}$. Con $100 \mathrm{~L} \mathrm{ha}^{-1}$ no obtuvo diferencias entre las muy finas producidas por las boquillas de cono hueco hasta las muy gruesas producidas por boquillas de abanico plano de aire inducido. Villalba et al. (2009) encontró que la cantidad de caldo que alcanzó a la parte inferior del cultivo fue mayor con gotas muy gruesas producidas por boquillas de abanico plano de aire inducido. Cunha et al. (2006) encontraron que a igualdad de volumen, las diferentes boquillas, depositaron la misma cantidad de producto independientemente del estrato analizado, sin diferencias en la eficacia de control de la roya y la productividad. El mismo autor (Cunha et al., 2011) evaluando boquillas de abanico plano deflector, doble abanico plano deflector, porta boquillas doble con boquillas abanico plano deflector y cono hueco no encontraron diferencias en la cantidad de azul brillante depositado en el estrato superior ni en el inferior, pero sí en el depósito entre los dos estratos. Ozkan et al. (2006) y Derksen et al. (2006) coinciden en que la utilización de gotas finas producidas con boquillas de cono hueco no promueve aumento en penetración, obteniendo mejores resultados con boquillas de abanico plano de gotas medias. Wolf \& Daggupati (2009) encontraron que la mayor cobertura en el estrato inferior del follaje fue logrado por gotas gruesas y medias producidas por diferentes tipos de boquillas de abanico plano, las boquillas simples tuvieron más penetración que las dobles, a pesar de esperar lo contrario. Klein \& Golus (2010), también obtuvieron mayor cobertura en la parte inferior del follaje con boquillas de abanico plano deflector con gotas gruesas y muy gruesas. La variación de las condiciones atmosféricas durante el día puede influenciar la eficacia de los tratamientos. Boller et al. (2011), encuentran que el cambio de angulación de las hojas de soja durante el día puede mejorar el control de enfermedades por facilitar la penetración de las gotas. Concordantemente Costa (2009) encuentra mejor control en aplicaciones al inicio de la tarde. Otro aspecto de interés es el efecto de la velocidad de aplicación sobre la penetración y la eficacia de los tratamientos. Zande et al. (2004), determinaron mayor deriva con el aumento de la velocidad de aplicación de 6 a $12 \mathrm{~km} \mathrm{~h}^{-1}$, aunque ese aumento fue menor con gotas de mayor tamaño. Resultados similares fueron obtenidos por Derksen et al. (2006) cuando evaluó la penetración y deriva con gotas finas y velocidades de 5 a $10 \mathrm{~km} \mathrm{~h}^{-1}$. Si bien el método de aplicación puede determinar diferencias de deposición, cobertura y penetración, las diferencias en el control de insectos son menos evidentes. Prado et al. (2010), encontraron diferencias entre parcelas tratadas y testigo sin tratar, pero mínimas diferencias entre tratamientos tanto en control de lagartas y chinches como en productividad y poder germinativo. Fiorin et al. (2011), obtuvieron mejor control de chinches y productividad del cultivo de soja con boquillas de aire inducido de gotas muy gruesas y aplicación aérea de bajo volumen oleoso (BVO) frente a la aplicación aérea convencional sin adyuvantes.

Los objetivos de los ensayos realizados fueron la evaluación del efecto del tamaño de gotas, la velocidad de aplicación y el momento de aplicación en la distribución del producto y el control de insectos en el cultivo de soja.

\section{Material y Metodos}

Los ensayos se realizaron en la localidad de Ombúes de Lavalle en el departamento de Colonia, Uruguay. El predio se encuentra ubicado a $34^{\circ} 03^{\prime} 55^{\prime \prime}$ Sur, $57^{\circ} 55^{\prime} 57^{\prime \prime}$ Oeste y 91 $\mathrm{m}$ de altura sobre el nivel del mar. La clasificación climática según Köppen corresponde a un clima templado lluvioso con ausencia de período seco y temperatura media del mes más cálido superior a $22^{\circ} \mathrm{C}(\mathrm{Cfa})$. La precipitación media es de 1200 $\mathrm{mm}$ anuales. La variedad utilizada fue Don Mario 3700 de grupo de madurez III sembrada a $0,34 \mathrm{~m}$ entre filas. La población en el momento del ensayo era de 437.000 plantas por hectárea.

Todas las aplicaciones se realizaron con un pulverizador autopropulsado marca Agroflex con boquillas distanciadas a 0,35 y $27 \mathrm{~m}$ de ancho de trabajo.

\section{Ensayo 1. Control de epinotia}

El ensayo fue realizado el 19 de enero de 2007. El objetivo específico fue evaluar el efecto del tamaño de gotas, momento y velocidad de aplicación, en la distribución del pulverizado y el control de epinotia (Epinotia aporema Walsingham). Se utilizó Metoxifenoxide (Intrepid ${ }^{\circledR} \mathrm{SC}$ ) a razón de $0,2 \mathrm{~L} \mathrm{ha}^{-1}$ 
de producto comercial, insecticida regulador de crecimiento simulador de la ecdisona con modo de acción por ingestión y con una tasa de aplicación de $80 \mathrm{~L} \mathrm{ha}^{-1}$. El cultivo se hallaba en el estado fenológico V8 según escala de Fehr y Caviness (Fehr \& Caviness, 1977). La altura promedio del cultivo era de $0,6 \mathrm{~m}$.

El diseño experimental fue de parcelas al azar y arreglo factorial de los tratamientos. El tamaño de las parcelas fue de 13,5 m de ancho y $50 \mathrm{~m}$ de largo. Los factores evaluados fueron: momento de aplicación, tamaño de gotas y velocidad de aplicación además de un testigo sin tratar y el tratamiento comercial del productor con gotas finas $(2 \times 2 \times 2+1+1)$. La eficacia biológica de los tratamientos se evaluó a través del conteo de larvas vivas de epinotia. Para ello se extrajo una muestra al azar de 15 plantas de cada una de tres estaciones de muestreo prefijadas realizando el conteo en laboratorio. Los resultados se expresaron en larvas cada 15 plantas.

La densidad de impactos sobre el follaje se determinó gracias al agregado al caldo del trazador fluorescente Blankophor 267\%®, (LANXESS AG, Leverkusen, Germany) con una concentración del $1 \%$. Se fijaron cinco estaciones de muestreo por parcela, en cada una se extrajeron cinco folíolos al azar del estrato superior y cinco del estrato medio del cultivo. El conteo en el haz y en el envés de los folíolos se realizó bajo microscopio estereoscópico con lámparas de luz negra en cinco conteos al azar en el haz y en el envés por folíolo. En la Figura 1 se observa la fotografía de un folíolo utilizado para el conteo de impactos.

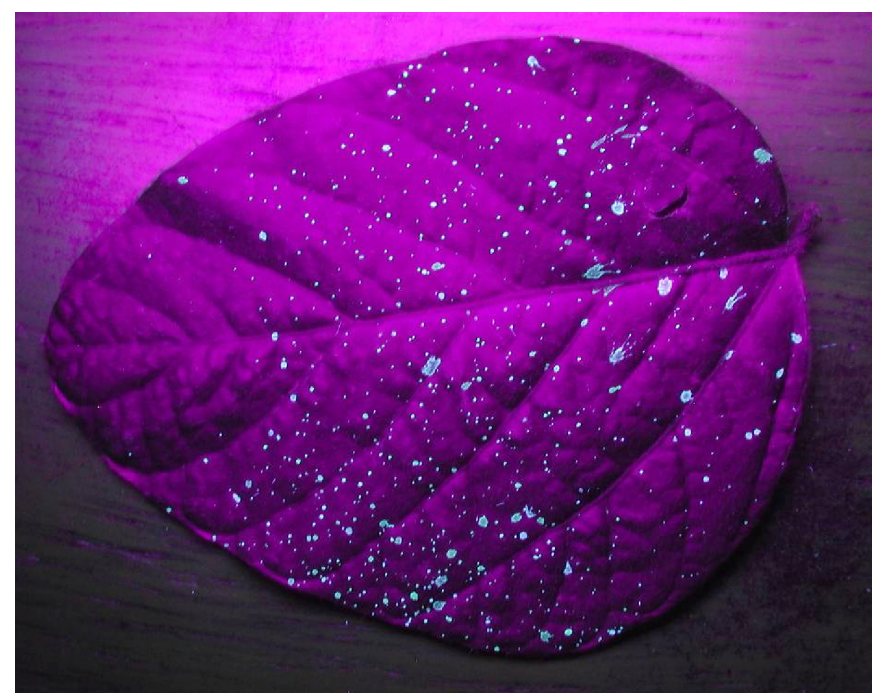

Figura 1. Fotografía de un folíolo bajo luz negra

También fue determinada la densidad de impactos sobre tarjetas hidrosensibles. Para ello se colocaron cinco soportes de madera por parcela a la altura máxima del cultivo, fijando una tarjeta por soporte. El conteo se realizó bajo microscopio estereoscópico en laboratorio mediante cuadrícula de $1 \mathrm{~cm}^{2}$, realizando 5 conteos por tarjeta. En la Tabla 1 se detallan los tratamientos evaluados.

Las condiciones meteorológicas durante el ensayo fueron determinadas mediante la colocación de dos termos higrógrafos digitales (modelo H8, Hobo, Bourne, MA) y un anemómetro de turbina (modelo LCA 6000, Davis Instruments, Hayward, CA). Las condiciones meteorológicas registradas no fueron demasiado contrastantes entre los dos momentos de aplicación. Se observaron valores muy bajos de humedad y velocidad de viento elevada (Tabla 2).

Tabla 1. Tratamientos ensayo epinotia

\begin{tabular}{|c|c|c|c|c|c|}
\hline Momento & $\begin{array}{c}\text { Velocidad } \\
\left(\mathrm{km} \mathrm{h}^{-1}\right)\end{array}$ & $\begin{array}{l}\text { Tamaño } \\
\text { de gota }\end{array}$ & Boquilla & $\begin{array}{c}\text { Presión } \\
\text { (bar) }\end{array}$ & $\begin{array}{l}\mathrm{DMV}^{* *} \\
(\mu \mathrm{m})\end{array}$ \\
\hline Día & 12 & Media & XR11002VP & 1,5 & 300 \\
\hline Día & 20 & Media & TT11002VP & 4,0 & 300 \\
\hline Noche & 12 & Media & XR11002VP & 1,5 & 300 \\
\hline Noche & 20 & Media & TT11002VP & 4,0 & 300 \\
\hline Día & 12 & $\begin{array}{l}\text { Muy } \\
\text { gruesa }\end{array}$ & TT11002VP & 1,5 & 419 \\
\hline Día & 20 & $\begin{array}{l}\text { Muy } \\
\text { gruesa }\end{array}$ & Al11002VS & 4,0 & 510 \\
\hline Noche & 12 & $\begin{array}{l}\text { Muy } \\
\text { gruesa }\end{array}$ & TT11002VP & 1,5 & 419 \\
\hline Noche & 20 & $\begin{array}{l}\text { Muy } \\
\text { gruesa }\end{array}$ & Al11002VS & 4,0 & 510 \\
\hline Día ${ }^{\star \star \star}$ & 15 & Fina & TXA8002VK & 2,5 & 185 \\
\hline
\end{tabular}

* Según norma ASAE S-572 FEB04

** Tamaño de gotas según el fabricante (Teejet)

*** Tratamiento comercial

Tabla 2. Condiciones meteorológicas durante los ensayos

\begin{tabular}{|c|c|c|c|}
\hline & \multicolumn{2}{|c|}{ Ensayo epinotia } & \multirow{2}{*}{$\begin{array}{c}\text { Ensayo chinches } \\
\text { Mediodía } \\
(13: 30 \text { a } 15: 20)\end{array}$} \\
\hline & $\begin{array}{c}\text { Mediodía } \\
\text { (12:00 a 14:40 h) }\end{array}$ & $\begin{array}{c}\text { Anochecer } \\
(19: 27 \text { a } 20: 27 \text { h) }\end{array}$ & \\
\hline $\begin{array}{l}\text { Temperatura } \\
\text { Promedio }\left({ }^{\circ} \mathrm{C}\right)\end{array}$ & 28,9 & 24,2 & 25,2 \\
\hline $\begin{array}{l}\text { Humedad relativa } \\
\text { Promedio (\%) }\end{array}$ & 37,5 & 35,7 & 72,9 \\
\hline $\begin{array}{l}\text { Velocidad del viento } \\
\text { (rango) }\left(\mathrm{km} \mathrm{h}^{-1}\right)\end{array}$ & $14,5-22,5$ & $12,8-20,7$ & $19-27$ \\
\hline $\begin{array}{l}\text { Velocidad del viento } \\
\text { promedio }\left(\mathrm{km} \mathrm{h}^{-1}\right) \\
\end{array}$ & 14,9 & 16,2 & 22,4 \\
\hline
\end{tabular}

\section{Ensayo 2. Control de chinches}

Fue realizado el 15 de marzo 2007 en otra área del mismo cultivo. El objetivo específico fue evaluar el efecto del tamaño de gotas y la velocidad de aplicación en la distribución del pulverizado y el control de chinches (Nezara viridula L. y Piezodorus guildini W.). Se aplicó el insecticida Endosulfán (Thiodan ${ }^{\circledR} 35 \mathrm{CE}$ ) a razón de 1,25 $\mathrm{L} \mathrm{ha}^{-1}$ de producto comercial y una tasa de $100 \mathrm{~L} \mathrm{ha}^{-1}$ de caldo. El Endosulfán es un insecticida con modo de acción por contacto e ingestión. El cultivo se hallaba en el estado fenológico R5 con una altura promedio de $1,05 \mathrm{~m}$. Las condiciones de temperatura y humedad eran muy adecuadas para una aplicación aunque el viento era algo superior a lo deseado (Tabla 2).

El diseño experimental fue de parcelas al azar y arreglo factorial de los tratamientos con dos repeticiones y un testigo $\sin \operatorname{tratar}(3 \times 2+1)$. Los factores evaluados fueron tamaño de gota y velocidad de aplicación. La eficacia biológica de los tratamientos se evaluó a través del conteo de larvas y adultos colectados en tres repeticiones de 25 redadas con red entomológica por parcela. La densidad de impactos se determinó sobre el follaje según técnica descripta en ensayo anterior. En la Tabla 3 se detallan los tratamientos evaluados.

El análisis estadístico del número de insectos se realizó mediante el procedimiento GLIMIX (SAS V9.0). Se asumió 
Tabla 3. Tratamientos ensayo chinches

\begin{tabular}{clccc}
\hline $\begin{array}{c}\text { Velocidad } \\
\left(\mathbf{k m ~ h}^{-1}\right)\end{array}$ & $\begin{array}{c}\text { Tamaño } \\
\text { de gota }^{*}\end{array}$ & $\begin{array}{c}\text { Identificación } \\
\text { de boquillas }\end{array}$ & $\begin{array}{c}\text { Presión } \\
(\mathbf{b a r})\end{array}$ & $\begin{array}{c}\mathbf{D M V}^{* *} \\
(\boldsymbol{\mu} \mathbf{m})\end{array}$ \\
21 & Fina & TXA8002 & 2,5 & 185 \\
12 & Fina & TXA8002 & 7,0 & 160 \\
21 & Media & DG9502E & 2,5 & 349 \\
12 & Media & DG9502E & 7,0 & 233 \\
21 & Muy gruesa & Al11002 & 2,5 & 565 \\
\hline
\end{tabular}

* Según norma ASAE S-572 FEB04

** Tamaño de gotas según el fabricante (Teejet)

una distribución binomial negativa de los datos experimentales y función de enlace logarítmica. Diferencias entre medias con probabilidad de error tipo I mayores al 5\% fueron consideradas iguales según la prueba de Tukey-Kramer. El análisis estadístico de los impactos sobre las hojas y sobre las tarjetas hidrosensibles se realizó mediante el procedimiento GENMOD (SAS V9.0). Se asumió una distribución Poisson de los datos experimentales y función de enlace logarítmica. La comparación de medias se realizó mediante la prueba de comparación de razones de verosimilitud. Diferencias entre medias con probabilidad de error tipo I mayores al 5\% fueron consideradas iguales según la prueba $\chi^{2}$.

\section{Resultados y Discusion}

El análisis factorial (Tabla 4) no permitió detectar diferencias entre niveles para ninguno de los factores estudiados.

Tabla 4. Efecto del tamaño de gotas, velocidad y momento de aplicación en el $N^{\circ}$ de larvas de epinotia

\begin{tabular}{lcccc}
\hline \multirow{2}{*}{ Factor } & \multirow{2}{*}{ Nivel } & \multicolumn{3}{c}{ Día } \\
\cline { 3 - 5 } & Muy Gruesa & $\mathbf{4}$ & $\mathbf{7}$ & $\mathbf{1 4}$ \\
\multirow{2}{*}{ Tamaño de gota } & $2,9 \mathrm{~ns}$ & $1,0 \mathrm{~ns}$ \\
& Medias & 7,3 & 4,7 & 0,7 \\
\hline \multirow{2}{*}{ Velocidad $\left(\mathrm{km} \mathrm{h}^{-1}\right)$} & 12 & 6,8 & 4,2 & 1,3 \\
& 20 & 5,7 & 3,4 & 0,3 \\
\hline \multirow{2}{*}{ Momento } & Día & 6,5 & 3,6 & 0,8 \\
& Noche & 6,0 & 4,0 & 0,8 \\
\hline
\end{tabular}

ns - sin diferencias significativas

La ausencia de efectos principales en el control de Epinotia coincide con los resultados de Prado et al. (2010), quienes tampoco encontraron diferencias en control de lagartas y chinches ni en productividad del cultivo.

En los conteos del día 4 después de la aplicación de los tratamientos se registra una disminución generalizada de la presencia de larvas de epinotia para casi todos los tratamientos con diferencias significativas con el testigo. Las mismas tendencias se observan en la evaluación a los 7 y 14 días (Tabla 5).

El análisis de las tarjetas hidrosensibles refleja adecuadamente lo esperable en función del tamaño de gotas utilizado (Tabla 6) con una relación inversa entre el tamaño de gotas y la densidad de impactos.

La deposición de gotas sobre las hojas (Tabla 7), muestra diferencias menores en la densidad de impactos lograda con los diferentes tamaños de gota utilizados que las tarjetas sensibles, representando más fielmente lo que sucede en el cultivo.

El tratamiento representativo de la aplicación comercial con gota fina logra densidad de impactos similar a los tratamientos
Tabla 5. Evolución de la cantidad de larvas de epinotia

\begin{tabular}{|c|c|c|c|c|c|c|}
\hline \multirow{2}{*}{$\begin{array}{l}\text { Tamaño } \\
\text { de gota }\end{array}$} & \multirow{2}{*}{$\begin{array}{l}\text { Horario de } \\
\text { aplicación }\end{array}$} & \multirow{2}{*}{$\begin{array}{c}\text { Velocidad } \\
\mathrm{km} \mathrm{h}^{-1}\end{array}$} & \multicolumn{4}{|c|}{ Día } \\
\hline & & & 0 & 4 & 7 & 14 \\
\hline Muy Gruesa & Día & 12 & $13^{*}$ & $5,0 \mathrm{~b}^{* *}$ & $1,6 \mathrm{~b}$ & $1,0 \mathrm{~b}$ \\
\hline Muy Gruesa & Noche & 12 & 7 & $5,3 \mathrm{~b}$ & $3,0 a b$ & $2,3 a b$ \\
\hline Muy Gruesa & Día & 20 & 12 & $7,7 a b$ & $6,0 a b$ & $0,7 \mathrm{~b}$ \\
\hline Muy Gruesa & Noche & 20 & 5 & $2,7 b$ & $1,0 \mathrm{~b}$ & $0,0 \mathrm{~b}$ \\
\hline Media & Día & 12 & 10 & $8,7 a b$ & $5,3 a b$ & $1,3 \mathrm{~b}$ \\
\hline Media & Noche & 12 & 10 & $8,3 a b$ & $6,6 a b$ & $0,7 \mathrm{~b}$ \\
\hline Media & Día & 20 & 8 & $4,7 \mathrm{~b}$ & $1,3 b$ & $0,3 \mathrm{~b}$ \\
\hline Media & Noche & 20 & 10 & $7,7 a b$ & $5,3 a b$ & $0,3 \mathrm{~b}$ \\
\hline Fina & Día & 15 & 5 & $5,7 \mathrm{~b}$ & $3,0 a b$ & $0,0 \mathrm{~b}$ \\
\hline Testigo & - & - & 8 & $20,0 \mathrm{a}$ & $11,0 \mathrm{a}$ & $9,7 \mathrm{a}$ \\
\hline
\end{tabular}

* Monitoreo previo sin repeticiones

** Medias de columnas identificadas con igual letra no difieren entre sí $(p \leq 0,05)$

Tabla 6. Densidad de impactos $\left(\mathrm{N}^{\mathrm{O}} \mathrm{Cm}^{-2}\right)$ en tarjetas hidrosensibles en ensayo epinotia

\begin{tabular}{llcc}
\hline $\begin{array}{c}\text { Tamaño } \\
\text { de gota }\end{array}$ & $\begin{array}{c}\text { Horario de } \\
\text { aplicación }\end{array}$ & $\begin{array}{c}\text { Velocidad } \\
\left(\mathbf{( k m ~ h}^{-1} \mathbf{)}\right.\end{array}$ & $\begin{array}{c}\text { Impactos } \\
\mathbf{( \mathbf { c m } ^ { - 2 } \mathbf { ) }}\end{array}$ \\
Muy Gruesa & Día & 12 & $33,1 \mathrm{ab}$ \\
Muy Gruesa & Noche & 12 & $49,0 \mathrm{~b}$ \\
Muy Gruesa & Día & 20 & $28,6 \mathrm{a}$ \\
Muy Gruesa & Noche & 20 & $28,2 \mathrm{a}$ \\
Media & Día & 12 & $77,1 \mathrm{c}$ \\
Media & Noche & 12 & $94,7 \mathrm{~d}$ \\
Media & Día & 20 & $98,4 \mathrm{~d}$ \\
Media & Noche & 20 & $94,4 \mathrm{~cd}$ \\
Fina & Día & 15 & $153,4 \mathrm{e}$ \\
\hline * Medias de columnas identificadas con igual letra no difieren entre sí $(\mathrm{p} \leq 0,05)$
\end{tabular}

de gotas medias en el estrato superior. La llegada de gotas al envés fue notoriamente reducida, siendo casi nula en el estrato medio. En la tabla 7, se observa que salvo en un caso, la deposición en el haz fue mayor en la noche que en el día ocurriendo lo contrario en el envés. La Figura 2 representa esa interacción llamativa. Esto concuerda con Boller et al. (2011), que obtienen mayor rendimiento de soja con aplicaciones al mediodía y principio de la tarde en comparación con las de la mañana, atribuyendo este hecho a mayor penetración por cambios en la orientación del follaje a lo largo del día. Esta misma razón podría explicar el mayor control de oídio en soja obtenido por Costa (2009), en aplicaciones al inicio de la tarde.

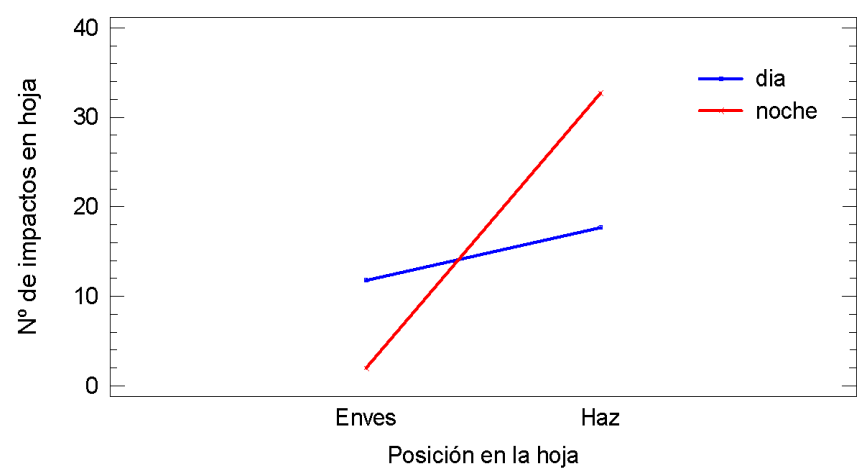

Figura 2. Densidad de impactos obtenida en el haz y en el envés de las hojas en diferentes momentos del día

El análisis factorial (Tabla 8) permitió detectar diferencias provocadas tanto por el tamaño de gotas utilizado como por la velocidad y momento de aplicación. 
Tabla 7. Densidad de impactos $\left(\mathrm{N}^{\circ} \mathrm{cm}^{-2}\right)$ en hojas de soja en ensayo epinotia

\begin{tabular}{|c|c|c|c|c|c|c|c|c|}
\hline \multirow{2}{*}{$\begin{array}{l}\text { Tamaño de } \\
\text { gota }\end{array}$} & \multirow{2}{*}{ Momento } & \multirow{2}{*}{$\begin{array}{c}\text { Velocidad } \\
\mathrm{km} \mathrm{h}^{-1}\end{array}$} & \multicolumn{3}{|c|}{ Estrato superior } & \multicolumn{3}{|c|}{ Estrato medio } \\
\hline & & & Haz & Envés & Suma & Haz & Envés & Suma \\
\hline Muy gruesa & Noche & 12 & $14,1 \mathrm{bc}$ & $15,3 d$ & $29,8 \mathrm{C}$ & $12,0 \mathrm{C}$ & $0,3 a$ & $12,3 b$ \\
\hline Muy gruesa & Noche & 20 & $19,3 \mathrm{c}$ & $0,1 \mathrm{a}$ & $19,4 \mathrm{~b}$ & $13,3 \mathrm{~cd}$ & $0,1 \mathrm{a}$ & $13,4 \mathrm{~b}$ \\
\hline Media & Día & 12 & $50,5 \mathrm{e}$ & $2,5 b$ & $53,0 \mathrm{~d}$ & $14,1 \mathrm{~cd}$ & $0,4 a b$ & $14,5 \mathrm{~b}$ \\
\hline Media & Noche & 12 & $72,9 f$ & $1,1 \mathrm{ab}$ & 74,0 ef & $49,0 f$ & $0,1 \mathrm{a}$ & $49,1 \mathrm{~d}$ \\
\hline Fina & Día & 15 & $24,3 \mathrm{c}$ & $41,3 \mathrm{f}$ & $65,6 \mathrm{e}$ & $33,0 \mathrm{e}$ & $0,1 \mathrm{a}$ & $33,1 \mathrm{c}$ \\
\hline
\end{tabular}

* Medias de columnas identificadas con igual letra no difieren entre sí $(p \leq 0,05)$

Tabla 8. Efecto del tamaño de gota, velocidad y momento de aplicación sobre la densidad de impactos $\left(\mathrm{N}^{\circ} \mathrm{cm}^{-2}\right)$ en ensayo epinotia

\begin{tabular}{|c|c|c|c|c|c|c|c|}
\hline \multirow{2}{*}{ Factor } & \multirow{2}{*}{ Nivel } & \multicolumn{3}{|c|}{ Estrato superior } & \multicolumn{3}{|c|}{ Estrato medio } \\
\hline & & Haz & Envés & Suma & Haz & Envés & Suma \\
\hline Tamaño de gota & Medio & $56,6 \mathrm{~b}$ & $1,8 \mathrm{a}$ & $58,4 \mathrm{~b}$ & $17,6 \mathrm{~b}$ & $0,3 \mathrm{a}$ & $18,0 \mathrm{~b}$ \\
\hline $\begin{array}{c}\text { Velocidad } \\
\left(\mathrm{km} \mathrm{h}^{-1}\right)\end{array}$ & 20 & $20,7 \mathrm{a}$ & $1,8 \mathrm{a}$ & $22,5 \mathrm{~b}$ & $8,0 \mathrm{~b}$ & $0,5 \mathrm{a}$ & $8,5 \mathrm{a}$ \\
\hline \multirow{2}{*}{ Momento } & Día & $17,2 \mathrm{a}$ & $11,7 \mathrm{~b}$ & $28,9 \mathrm{a}$ & $6,2 \mathrm{a}$ & $1,6 b$ & $7,8 \mathrm{a}$ \\
\hline & Noche & $34,2 \mathrm{~b}$ & $0,6 \mathrm{a}$ & $34,8 \mathrm{a}$ & $18,5 \mathrm{~b}$ & $0,1 \mathrm{a}$ & $18,6 \mathrm{~b}$ \\
\hline
\end{tabular}

* Medias de columnas identificadas con igual letra para cada factor no difieren entre sí $(p \leq 0,05)$

El análisis de varianza del número de impactos en hojas también permitió detectar diferencias muy significativas entre el número de impactos obtenido en el estrato superior y el estrato medio, así como los obtenidos entre el haz o el envés para ambos estratos (prob. $f \leq 0,001$ ). Los resultados coinciden con Antuniassi et al. (2004) y Galvez et al. (2005), quienes comparando diferentes tipos de boquillas en la cobertura de hojas de soja encontraron que las boquillas de gotas más finas tenían mejor cobertura en los estratos inferiores. También en forma consistente la cantidad de impactos fue mayor a la menor velocidad de aplicación. Debe tomarse con precaución ese resultado debido a que la utilización de las boquillas de aire inducido para lograr gotas muy gruesas a $20 \mathrm{~km} \mathrm{~h}^{-1}$ (Tabla 1) puede haber originado esa reducción.

Los trabajos de Derksen et al. (2006) y Zande et al. (2004) obtienen efectos similares de la velocidad de aplicación pero en ningún caso evaluando velocidades tan altas como las que son propias de los equipos autopropulsados actuales.

Otro resultado de especial consideración fue que los impactos en el envés en el estrato inferior fueron muy bajos en todos los casos demostrando la casi imposibilidad de alcanzar buenos valores de deposición de gotas a ese nivel.

\section{Ensayo chinches}

No se detectaron diferencias en número de chinches entre niveles para los factores tamaño de gotas ni velocidad de aplicación en las diferentes fechas. Los resultados en cuanto al efecto del tamaño de gotas utilizado para control de chinches coinciden en general con los obtenidos por Prado et al. (2010) y por Fiorin et al. (2011).

Tabla 9. Efecto del tamaño de gota y velocidad de aplicación en el número de chinches

\begin{tabular}{ccccc}
\hline \multirow{2}{*}{ Factor } & & \multicolumn{3}{c}{ N. de chinches } \\
\cline { 3 - 5 } & Gruesa & $5,2 \mathrm{~ns}$ & $2,0 \mathrm{~ns}$ & $1,0 \mathrm{~ns}$ \\
\multirow{3}{*}{ Tamaño gota } & Media & 4,5 & 2,1 & 1,0 \\
& Fina & 2,7 & 1,5 & 1,3 \\
\hline \multirow{2}{*}{ Velocidad $\left(\mathrm{km} \mathrm{h}^{-1}\right)$} & 12 & 4,3 & 2,4 & 1,1 \\
& 21 & 4,0 & 1,6 & 1,1 \\
\hline
\end{tabular}

ns - $\sin$ diferencias significativas

Tabla 10. Efecto de la velocidad y tamaño de gota en el número de chinches

\begin{tabular}{ccccc}
\hline \multirow{2}{*}{ Tamaño gota } & $\begin{array}{c}\text { Velocidad } \\
\left(\mathbf{k m ~ h}^{-1}\right)\end{array}$ & \multicolumn{3}{c}{ Día } \\
\cline { 3 - 5 } Gruesa & 12 & $\mathbf{0}$ & $\mathbf{7}$ & $\mathbf{9}$ \\
Gruesa & 21 & $5,2^{\mathrm{NS}}$ & $2,5 \mathrm{a}^{\mathbf{}}$ & $1,3^{\mathrm{NS}}$ \\
Media & 12 & 4,2 & $1,8 \mathrm{a}$ & 0,7 \\
Media & 21 & $4,0 \mathrm{a}$ & 1,0 \\
Fina & 12 & 4,0 & $1,7 \mathrm{a}$ & 1,0 \\
Fina & 21 & 1,5 & $1,7 \mathrm{a}$ & 0,7 \\
Testigo & - & 5,5 & $8,6 \mathrm{a}$ & 2,0 \\
\hline
\end{tabular}

* Medias de columnas identificadas con igual letra no difieren entre sí $(p \leq 0,05)$

Tabla 11. Densidad de impactos $\left(\mathrm{N}^{\circ} \mathrm{cm}^{-2}\right)$ en hojas en ensayo chinches

\begin{tabular}{|c|c|c|c|c|c|c|c|}
\hline \multirow{2}{*}{ Factor } & & \multicolumn{3}{|c|}{ Estrato superior } & \multicolumn{3}{|c|}{ Estrato medio } \\
\hline & & Haz & Envés & Suma & Haz & Envés & Suma \\
\hline Tamaño de gota & Media & $93,1 \mathrm{~b}$ & $42,6 \mathrm{~b}$ & $69,6 \mathrm{~b}$ & $61,6 \mathrm{~b}$ & $0,5 \mathrm{a}$ & $62,2 \mathrm{~b}$ \\
\hline \multirow{2}{*}{ Velocidad $\left(\mathrm{km} \mathrm{h}^{-1}\right)$} & 12 & $85,2 \mathrm{a}$ & $50,0 \mathrm{a}$ & $68,6 \mathrm{a}$ & $65,0 \mathrm{~b}$ & $0,9 \mathrm{a}$ & $66,2 a$ \\
\hline & 21 & $84,6 \mathrm{a}$ & $52,5 \mathrm{a}$ & $72,1 \mathrm{~b}$ & $48,1 \mathrm{a}$ & $0,9 a$ & $49,0 \mathrm{~b}$ \\
\hline
\end{tabular}

${ }^{\star}$ Medias identificadas en columnas con igual letra para cada factor no difieren significativamente entre sí con $(p \leq 0,05)$ 
No se detectaron diferencias entre tratamientos en el control de chinches salvo para el día 7 donde todos los tratamientos se diferenciaron del testigo sin tratar (Tabla 10).

En la Tabla 11 se observa que la cantidad de impactos sigue la relación inversa con el tamaño de gotas observándose mayor número de impactos con gotas finas tanto en el estrato superior como medio. Estas diferencias no se ven reflejadas en el control de chinches. El método de cuantificar los depósitos exclusivamente por medio de conteos de impactos suele no reflejar adecuadamente la distribución de la cantidad de producto debido al efecto del diferente tamaño de gotas. Al igual que en el ensayo realizado para control de epinotia, la densidad de impactos en el envés del estrato medio es casi nula.

\section{Conclusiones}

1. El control de epinotia y chinches obtenido con la utilización de gotas muy gruesas, medias y finas es similar, observándose solo diferencias entre las parcelas tratadas y no tratadas.

2. No se detectan efectos del momento ni de la velocidad de aplicación en el control de epinotia. El control de chinches tampoco fue afectado por la velocidad de aplicación.

3. Las diferencias en la densidad de impactos obtenidas sobre tarjetas hidrosensibles y sobre el follaje son básicamente determinadas por el tamaño de gotas utilizado.

4. En el estrato medio se observan menos impactos que en el superior, particularmente en el envés donde los impactos observados en todos los casos son muy bajos.

5 . El uso de gotas muy gruesas y medias se demostró eficaz en el control de insectos, siendo su utilización una herramienta de gran potencial en la disminución de la deriva por aplicaciones en el cultivo de soja frente a aplicaciones con gotas finas.

\section{Lteratura Citada}

Antuniassi, U. R.; Camargo, T.V.; Bonelli, M. A. P. O.; Romagnole, E. W. C. Avaliação da cobertura de folhas de soja em aplicações terrestres com diferentes tipos de pontas. In: Simpósio Internacional de Tecnologia de Aplicação de Agrotóxicos, 3, 2004, Botucatu. Anais... Botucatu: FEPAF, 2004. CD-Rom

Barbosa, R. N.; Griffin, J. L.; Hollier, C. A. Effect of spray rate and method of application in spray deposition. Applied Engineering in Agriculture, v.25, p.181-184, 2009.

Boller, W; Cigana, M.; Costa, D. I. da. Condições do ar e angulação das folhas influenciam a qualidade das pulverizações na cultura da soja? Revista Plantio Direto, v.121, p.33-37, 2011.

Boschini, L.; Contiero, R. L.; Macedo Júnior, E. K.; Guimarães, V. F. Avaliação da deposição da calda de pulverização em função da vazão e do tipo de bico hidráulico na cultura da soja. Acta Scentiarum. Agronomy, v.30, p.171-175, 2008.

Costa, D. I. da. Eficiência e qualidade de aplicações de fungicidas, por vias terrestre e aérea, no controle de doenças foliares e no rendimento de grãos de soja e milho. Passo Fundo: UPF, 2009. 126p. Tese Doutorado
Cunha, J. P. A. R.; Reis, E. F.; Santos, R. O. Controle químico da ferrugem asiática da soja em função de ponta de pulverização e de volume de calda. Ciência Rural, v.36, p.1360-1366, 2006.

Cunha, J. P. A. R.; Silva, R. A. M.; Olivet, J. J. Avaliação de pontas e volumes de pulverização na aplicação de fungicida na cultura da soja (Glycine max (L.) Merrill). Revista de La Facultad de Agronomía, Universidad Del Zulia, v.28, p.44-360, 2011.

Derksen, R. C.; Zhu, H.; Ozkan, H. E.; Dorrance, A. E.; Krause, C. Effects of air assisted and conventional spray delivery systems on management of soybeans diseases. Aspects of Applied Biology, v.77, p.415-422, 2006.

Fehr, W. R.; Caviness, C. E. Stages of soybean development. Iowa: Iowa State University: Cooperative Extension Service Special Report 80, 1977. 12p

Fiorin, R. A.; Stürmer, G. R.; Carús, J. V.; Costa, I. F. D. da; Perini, C. R. Métodos de aplicação e inseticidas no controle de percevejos na cultura da soja. Ciências Agrárias, v.32, p.139-146, 2011.

Galvez, M. R.;Vinciguerra, H. F.; Rodriguez,W.; Sabate, S.; Soldini, E. A.; Devani, M. R.; Olea, I. L.; Ploper, L. D. Evaluación de la penetración del asperjado producido por diferentes boquillas en aplicaciones terrestres orientadas al control de la roya de la soja. Tucumán: Estación Experimental Obispo Colombres. 2005. 12p. Publicación especial n.27.

Hanna, H. M.; Robertson, A. E.; Carlton, W. M.; Wolf, R. E. Nozzle and carrier application effects on control of soybean leaf spot diseases. Applied Engineering in Agriculture, v.25, p.5-13, 2009.

Klein, R.; Golus, J. Evaluation of soybean (Glycine max) canopy penetration with several nozzle types and pressure. In: International Advances in Pesticide Application, 2006, Cambridge. Porceedings... Cambridge: Robinson College, 2010. p.35-39.

Ozkan, H. E.; Zhu, H.; Derksen, R. C.; Guler, H.; Krause, C. R. Evaluation of various spraying equipment for effective application of fungicides to control Asian Soybean Rust. Aspects of Applied Biology, v.77, p.423-431, 2006.

Prado, E. P.; Raetano, C. G.; Aguiar Júnior, H. O.; Christovam, R. S.; Pogetto, M. H. F. A. dal; Gimenes, M. J. Velocidade do fluxo de ar em barra de pulverização no controle químico de Anticarsia gemmatalis, Hübner e percevejos na cultura da soja. Bragantia, v.69, p.995-1004, 2010.

Villalba, J.; Martins, D.; Costa, N. V.; Domingos, V. D. Deposição da calda de pulverização em cultivares de soja no estádio R1. Ciência Rural, v.39, p.1738-1744, 2009.

Wolf, R. E.; Daggupati, N.P. Nozzle type effect on soybean canopy penetration. Applied Engineering in Agriculture, v.25, p.2330, 2009.

Zande, J. C. van de; Stallinga, H.; Michelsen, J. M. P. G.; Velde, P. van. Effect of sprayer speed on spray drift. In: International Conference on Pesticide Application for Drift Management, 2004, Waikoloa. Proceedings... Pullman: Washington State University, 2004. p.339a-339j. 